\title{
BNCI Horizon 2020 - Towards a Roadmap for Brain/Neural Computer Interaction
}

\author{
Clemens Brunner ${ }^{1}$, Benjamin Blankertz ${ }^{2}$, Febo Cincotti ${ }^{3}$, Andrea Kübler ${ }^{4}$, \\ Donatella Mattia $^{3}$, Felip Miralles ${ }^{5}$, Anton Nijholt ${ }^{6}$, Begonya Otal ${ }^{5}$, \\ Patric Salomon ${ }^{7}$, and Gernot R. Müller-Putz ${ }^{1}$ \\ ${ }^{1}$ Institute for Knowledge Discovery, Graz University of Technology, Graz, Austria \\ ${ }^{2}$ Chair for Neurotechnology, Berlin Institute of Technology, Berlin, Germany \\ ${ }^{3}$ Neuroelectrical Imaging and BCI Lab, Fondazione Santa Lucia, Rome, Italy \\ ${ }^{4}$ Institute of Psychology, Biological Psychology, Clinical Psychology and Psychotherapy, \\ University of Würzburg, Würzburg, Germany \\ ${ }^{5}$ Barcelona Digital Technology Centre, eHealth R\&D, Barcelona, Spain \\ ${ }^{6}$ Department EWI, Research Group Human Media Interaction, \\ University of Twente, Enschede, The Netherlands \\ ${ }^{7}$ EnablingMNT GmbH, Berlin, Germany \\ clemens.brunner@tugraz . at
}

\begin{abstract}
In this paper, we present BNCI Horizon 2020, an EU Coordination and Support Action (CSA) that will provide a roadmap for brain-computer interaction research for the next years, starting in 2013, and aiming at research efforts until 2020 and beyond. The project is a successor of the earlier EUfunded Future BNCI CSA that started in 2010 and produced a roadmap for a shorter time period. We present how we, a consortium of the main European $\mathrm{BCI}$ research groups as well as companies and end user representatives, expect to tackle the problem of designing a roadmap for BCI research. In this paper, we define the field with its recent developments, in particular by considering publications and EU-funded research projects, and we discuss how we plan to involve research groups, companies, and user groups in our effort to pave the way for useful and fruitful EU-funded BCI research for the next ten years.
\end{abstract}

Keywords: Brain-computer interfaces, Horizon 2020, roadmap, Future BNCI, mental state monitoring, BCI publications, EU projects, BCI Society, usercentred design, ethical guidelines.

\section{Introduction}

Brain-computer interfaces (BCIs) have become a popular topic for research in recent years. A BCI is a communication device which allows people to control applications through direct measures of their brain activity. A BNCI (brain/neuronal computer interaction) system extends a BCI by including other physiological measures such as muscle or eye movement signals. The number of BCI research groups around the world, peer-reviewed journal articles, conference abstracts, and attendance at relevant 
conferences are indicators of the rapid growth of this field. With dozens of companies and research groups actively participating in the development of BCIs and related technologies, collaboration, a common terminology, and a clear roadmap have become important topics. To provide a solution to these issues, the European Commission (EC) funded the coordination action Future BNCI in 2010/2011. This project was the first effort to foster collaboration and communication among key stakeholders. Furthermore, this project created a five-year roadmap for BCI research. In 2013, the EC decided to continue the efforts initiated by Future BNCI by funding the development of a more long-term roadmap within the new framework program Horizon 2020.

In this paper, we explain how BNCI Horizon 2020 will address and predict new developments in BNCI research, including new applications for people with motor, sensory, cognitive and mental disabilities, and people without these disabilities. The new roadmap will touch upon other key topics including ethics, societal needs for and acceptance of BNCI systems, user-centered approaches, evaluation metrics, and the transfer of technology from research labs to the market. A clear and comprehensive roadmap produced by BNCI Horizon 2020 will lay the foundations for, and impact on, a (continued) dominance and clear visibility of European research groups in the future. In addition, the roadmap will display opportunities, but also limitations and constraints, for the industrialization and commercialization of BNCIs. The aim of this paper is to inform the research community about this project and invite comments and involvement.

\section{$2 \quad$ BCIs and BNCIs Definitions}

According to the most commonly used definition, a brain-computer interface (BCI) is a communication device that classifies brain activity and controls a device such as a spelling application $[1,2,3,4]$, a neuroprosthesis [5,6], a domestic environment [7,8], a wheelchair [9,10], a telepresence robot[11,12], an internet browser [13,14], computer games $[15,16,17,18]$, or creative expression $[19,20]$. A BCI uses signals directly recorded from the brain, operates online, provides feedback, and relies on goal-directed behavior [21,22]. A more recent definition describes a BCI as follows [23]: “A BCI is a system that measures central nervous system (CNS) activity and converts it into artificial output that replaces, restores, enhances, supplements, or improves natural CNS output and thereby changes the ongoing interactions between the CNS and its external or internal environment." This definition includes BCIs that do not require intentional control, which are sometimes referred to as passive BCIs [24]. Other related terms include affective BCIs [25], emotional BCIs [26] or mental state monitors [27,28]. Figure 1 depicts the scheme of a BCI and lists possible applications.

The term brain/neuronal computer interaction (BNCI) was introduced by the European Commission (EC) without a clear definition at the beginning. However, the research community has adopted the convention that a BNCI differs from a BCI only in the used signals. Specifically, a BNCI does not only rely on direct measurement of brain activity, but also includes signals from other physiological activity such as eye 


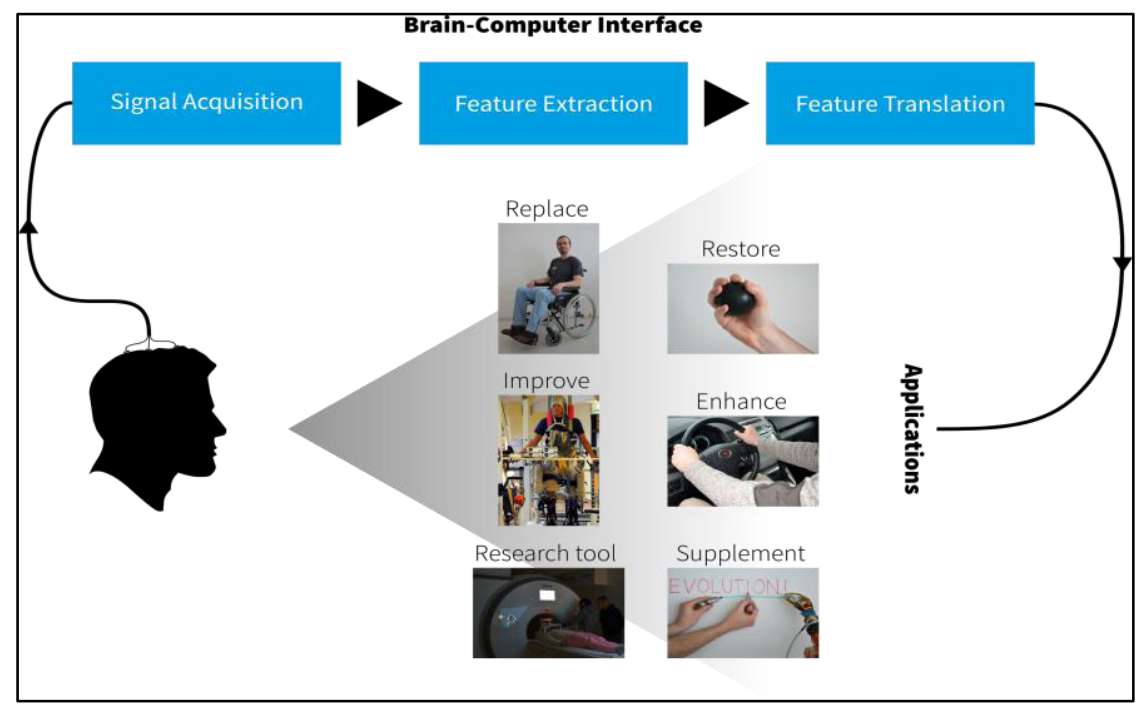

Fig. 1. BCI scheme and possible application scenarios (adapted from [23])

movement (electro-oculogram, EOG), muscle activity (EMG, electromyogram), or heart rate (HR, derived from the electrocardiogram, ECG). Furthermore, a device that combines a BCI with another input device is also known as a hybrid BCI $[29,30]$ or a multimodal BCI [31].

\section{BCI and BNCI Research Field}

Research on BNCIs and BCIs started in the 80s of the last century (although first ideas date back to Vidal et al. in the 70s) and is currently transforming from a small field in its infancy to a large research effort across the globe. The number of scientific publications in this field is steadily increasing every year (see Figure 2). Another measure of progress is the attendance at international conferences; for example, the five Graz BCI conferences (organized by Graz University of Technology in Graz, Austria) in 2002, 2004, 2006, 2008, and 2011 were attended by 32, 52, 95, 116, and 236 people, respectively. The 5th International BCI Meeting in Pacific Grove, CA, USA, in June 2013 attracted more than 300 attendees.

While BCI research traditionally focused almost exclusively on applications for patients, the interest in developing non-medical applications has been growing recently; this targets a much larger user group. These applications include BCIs to control games [16,17], but also a plethora of other novel approaches are investigated [32]. EEG-based monitoring of workload can be used for example to mitigate workload in the case of overload in car/truck/train driving or aviation [33]. Similarly, monitoring the level of attention can be used to initiate counter-measures and thereby help to avoid hazardous situations in industrial workplaces [34,28]. Electrophysiology can 


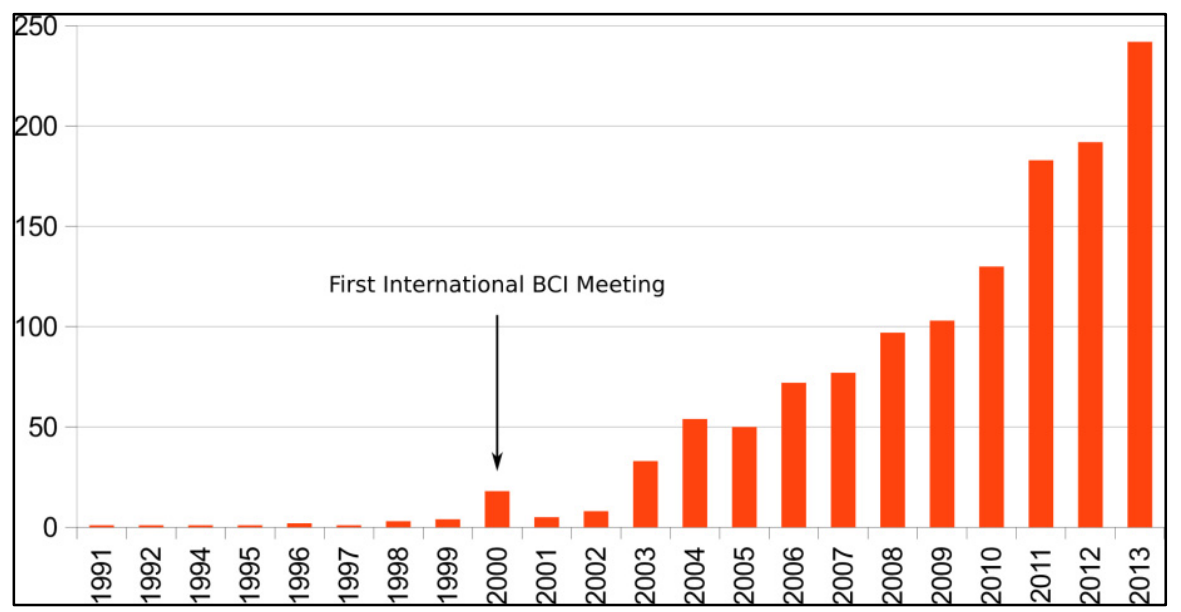

Fig. 2. Number of BCI publications per year as obtained by a PubMed query "brain-computer interface" in indexed English articles queried on 01/14/2014

also be exploited to detect precursors of braking intention and gives rise to a neuroergonomic approach to driving assistance [35]. Furthermore, many opportunities exist to improve human-computer interaction [24] using BCI systems by resorting to implicit information in the perceptual and cognitive processes. Examples include approaches based on (a) the possibility to extract subconscious processes from the EEG as exemplified in the detection of distortions in audio samples [36], (b) the EEGbased recognition of unexpected events [37], and (c) the quantification of the depth of processing [38].

Numerous companies are now active in the BCI sector. The roadmap developed in the EU-funded coordination action (CA) Future BNCI lists 39 companies working in and producing BCIs or related devices for different market sectors such as health and neurofeedback, assistive technology, education, safety and security, entertainment and performance, research, and financial and marketing. Within BNCI Horizon 2020, we have already identified more than 100 potential companies either directly developing BCIs or related devices, or aiming to integrate BCI-based technology into their product portfolio or upcoming market applications.

Several sources also indicate that commercial interest in BNCI research is increasing. Guger Technologies, a major supplier of amplifiers and other equipment for BNCI research, reports an increase in annual sales for BCI equipment of about $35 \%$ per annum since 2005. Other companies such as NeuroSky, Emotiv, InterAxon, and OCZ have heavily publicized new BNCIs for game control that did not exist a few years ago. These toys use very simple, inexpensive, sometimes single-channel systems aimed at healthy users for entertainment - instead of complex, expensive, often multi-channel systems aimed at severely disabled users for assistive technology. NeuroSky sold over one million chips in 2011 for BCI toys like the Necomimi Cat Ears and Star Wars Force Trainer [39]. Other companies like Advanced Brain Monitoring, Neuroelectrics, NeuroFocus and EmSense have developed more expensive BCIs that 
are also aimed at healthy users. These systems include devices to monitor alertness, sleep or anticipation. Healthy users seem to be a rapidly growing market for BNCIs. However, it is unclear which users, applications, companies or usage environments will be the strongest to emerge soon.

\section{Relevant European Research Projects}

The EU has funded several projects dealing with BNCIs within its Framework Programmes FP6 and FP7. Thirteen projects are part of a BNCI cluster within the eInclusion initiative, which consists of the following projects: TOBI (11/08-01/13), TREMOR (09/08-04/10), BRAIN (09/08-08/11), DECODER (02/10-04/13), BETTER (02/10-01/13), BrainAble (01/10-12/12), Future BNCI (01/10-12/11), AsTeRICS (01/10-12/12), MINDWALKER (01/10-12/12), MUNDUS (03/10-02/13), ABC (11/11-10/14), BackHome (01/12-06/15), and WAY (10/11-09/14). CONTRAST (11/11-10/14) and MindSee (09/13-08/16) are other ongoing EU-funded projects dealing with BCIs outside the BNCI cluster. Here, BCIs are used for feedback of EEG activity to improve general and specific cognitive functions (such as attention and inhibition) in stroke patients. Moreover, several researchers received individual European or national grants. Among these projects, Future BNCI was the first coordination action (CA) in this research field. Its main goals were (1) to foster collaboration between BCI researchers, (2) to develop a common terminology, and (3) to identify roadmaps and opportunities for the field of BCI research. The integrated project (IP) TOBI took over and continues to develop parts of the outcome of Future $\mathrm{BNCI}$, in particular the roadmap.

\section{Current Challenges}

The field of BNCI research is growing rapidly, and there are no major coordination efforts in place to ensure efficient communication and collaboration between key stakeholders. For example, the field has still neither agreed upon common definitions of key terms, nor upon a common procedure concerning ethical issues, and opinions on promising future directions differ vastly. The fact that stakeholders address these issues individually not only wastes precious resources, but also translates to an excess expenditure, which could be reduced by a close and supported collaboration.

The synergy in efforts appears crucial to foster the translation of several BNCI research developments from the laboratories to real world scenarios, which include healthy and disabled end users [40,41].

The previous CA Future BNCI has successfully started to address these critical issues. However, these efforts were only the first steps toward our goal to identify and address current and future challenges and to create a coordinated research field including the foundation of a BCI Society, which would be a strong indicator of the maturation of the field.

On the whole, four major challenges can be identified: (1) The growing need for standards is still unmet. This challenge entails many aspects of standardization: terms 
and definitions for BCIs and BNCIs, guidelines for reporting key details of BCI articles, ethical guidelines for working with patients, and certifications for relevant personnel. There is still confusion and disagreement about what constitutes a BCI or BNCI. (2) There is inadequate interaction within the BNCI community. This makes it difficult to work together on standards, and also impedes collaboration for research and development. There is no central society or group that represents the BCI community and can foster interaction with policy makers, other stakeholders, the media and other groups. (3) There is inadequate dissemination outside of the BNCI community. Most doctors, patients, students, and the general public do not know about BCIs, or have unrealistic views based on how the technology is presented in the media and some news sources. Other research and development communities, particularly involving assistive technologies and patient care, do not interact enough with BNCI researchers. (4) There is little agreement on the most promising future directions. Since integrating BCIs with other BNCIs, other interaction paradigms, and other emerging technologies entails working with new people and new groups, the lack of interaction has made it difficult to objectively assess which technologies (and new combinations thereof) are promising. Companies, policy makers, medical decision makers, patients, and other groups without a BNCI background may find it especially difficult to identify the most promising products, directions, funding opportunities, and treatment options.

These four main challenges cannot be addressed without an effective coordination effort. It seems unlikely that any project can align constituencies and prepare future joint research and roadmaps when the relevant disciplines and stakeholders are not even clearly identified. The principal vision of BNCI Horizon 2020 is to address these challenges by establishing and supporting a thriving, efficient, and well-connected BNCI community.

\section{Goals and Objectives}

With BNCI Horizon 2020, we continue coordination efforts that were initiated by Future BNCI to ensure that progress is not impeded by a lack of infrastructure, lack of communication between key stakeholders, ambiguous terminology or an unclear roadmap of the research field. BNCI Horizon 2020 will develop a roadmap document, building on the results of Future BNCI and TOBI, but also on results of other past and current projects. The roadmap will serve as a guideline and will contain conclusions, recommendations, and a provision of ideas for future activities that will be supported by the EU framework program Horizon 2020. In the roadmap, we will identify new BNCI opportunities and synergies with existing fields, with respect to applications for people-with motor, sensory, and cognitive disabilities as well as for healthy users. We plan to identify these opportunities from a research, industrial, and end users' perspective.

The end users' perspective will embrace the principles of the user-centered design to address some of the future priorities in terms of BNCI applications in real world 
medical (i.e., assistive and rehabilitation field) and non-medical scenarios (i.e., entertainment and general purpose in healthy people).

The user-centered design was standardized in ISO-DIS-9241-210. It is focused on the concept of usability, i.e. "the extent to which a product can be used by specified users to achieve specified goals with effectiveness, efficiency and satisfaction in a specified context of use". The principles of this approach are adopted as an iterative process in the development of assistive technology (AT) devices as well as in the Human-Computer Interaction field, addressing the understanding and specification of users' needs and the context of use, and evaluation against the defined requirements.

In recent years, development of communication systems based on BNCI technology has started to take advantage of the AT design principles $[18,19,41,42]$. BNCI Horizon 2020 will capitalize on previous and current research and application experience to define standards for the efficient development and assessment of BNCIbased technologies taking in to account users' requirements and needs.

BNCI Horizon 2020 will encourage discussion and collaboration; the project will also disseminate knowledge and information among BCI researchers and the general public. Our main communication and dissemination channels are (a) workshops and conferences where we explain the aims of BNCI Horizon 2020 and collect feedback; (b) a dedicated community website, based on and incorporating information from the Future BNCI website (future-bnci.org), that will not only present results from the project, but will be a platform for BNCI research in general and an information hub and community portal for researchers, reviewers, industrial partners, and the general public at large; (c) the creation of an open access database of BCI data sets on our website to foster competition and reproducibility of results. In addition, BNCI Horizon 2020 will actively support the foundation of an international BCI Society.

\section{Coordination and Networking Efforts}

First and foremost, a major result of the project will be a concise roadmap, a document that will build on the roadmap produced by the Future BNCI and TOBI projects. We will incorporate new findings to be generated within BNCI Horizon 2020 through the knowledge of its partners, their background, links to other projects, and by engaging with the relevant stakeholders (end user representatives, industry, and research) through questionnaires, workshops, and interviews. Roadmapping will be driven by interactive and iterative activities.

Based on the existing Future BNCI roadmap, initial application scenarios will be developed. These scenarios will be verified through the advisory board and updated throughout the project. The current state of the art (in all three areas research, industry, and end users) for these scenarios will be extracted from the existing roadmaps and updated with the latest knowledge.

User requirements and visions will be assessed from/with end users (e.g., focus groups) and visionary stakeholders. Initially, we will capitalize on previously established networking between a large community of end users (individuals, representative associations, assistive technology solution providers, and medical staff) and national and EU research projects. These requirements will be discussed with research and industry to check what is possible in 5-10 years from their view, which is then to 
be agreed with industry in terms of what is realistic and commercially feasible within this timeframe.

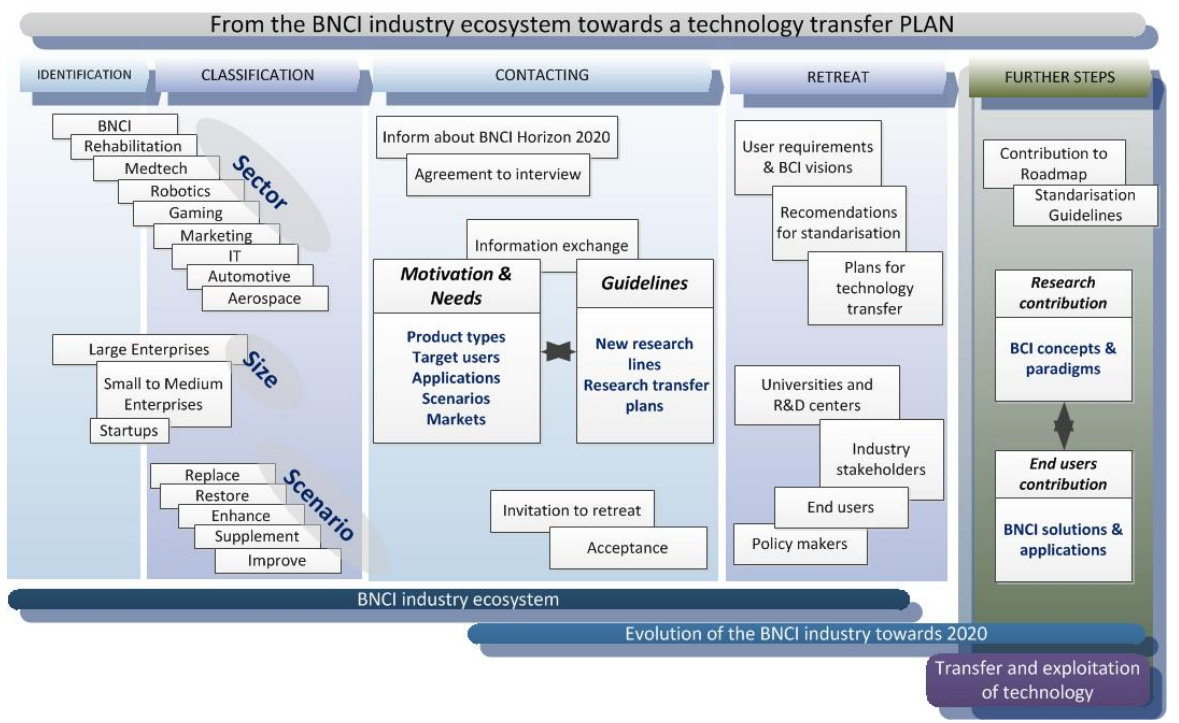

Fig. 3. From the industry ecosystem towards a technology transfer plan for the overall BNCI Horizon 2020 roadmap: coordination and networking efforts among academia, industry stakeholders, policy makers, and end users

The updated scenarios will again be discussed with end user representatives to verify if these ideas could actually add value for them and their stakeholders. The resulting technological challenges and the research needed to address them will then be assessed in joint discussions with industry and research representatives. Finally, a timeline can be drawn of technologies/research needed to address challenges and the possible results in terms of (commercial) products and services.

Additionally, we will collect and/or estimate data on numbers of users (EU- and world-wide) and calculate market data in terms of potential users of such devices to estimate the possible market and in general the possible impact of BCI technologies.

Other networking efforts include the organization of dedicated meetings with stakeholders, conferences, workshops, and special sessions.

Therefore, we will mainly focus on coordination and processing inputs from all identified industry stakeholders in BNCI and related domains to develop guidelines, recommendations, actions and plans about current and future products, application scenarios, markets and business models, with a strong focus on success stories, technology transfer, and exploitation avenues. We have been designing different strategies to acquire and process qualitative industry ecosystem data, and to manage a market analysis, in order to develop these guidelines and recommendations towards a technology transfer plan (see Figure 3).

Several steps prior to one of the main networking activities, the BNCI Horizon 2020 Retreat, correspond to the following coordination efforts: First, the identification of 
major industry stakeholders coming from the whole BNCI consortium. Second, the classification of those identified BNCI and new potential industry stakeholders from related domains. So far, we have already identified over 100 large enterprises, small to medium enterprises and startups, and defined the corresponding classification by company size, sector, location, potential BNCI scenarios, target users, and applications. Third, we have started to contact those main players and inform them about BNCI Horizon 2020 activities. Further, we try to engage them to participate by inviting them to the retreat and by asking them to answer questionnaires about their company in relation to BNCI motivations and needs. The first networking activity, the upcoming retreat, will enable us to engage in discussion sessions, surveys, and workshops. Thereafter, there will be a need to establish new procedures and methods to process all collected data from the research and academia contribution, the industry stakeholders' motivations and needs, and end users' new potential BNCI solutions and applications.

The overall BNCI Horizon 2020 roadmap will contain the analyzed material obtained from these major coordination and networking efforts. This will rely on qualitative market research, through the links to the industry, academia and end users coming from the whole consortium as well as from our advisory board and the upcoming BCI Society.

\section{Conclusions}

BNCI Horizon 2020 will impact the field of BNCIs in the following ways:

- We will provide a roadmap of the field of BNCIs for policy makers, politicians, researchers, companies, end user associations, end users, clinical staff, and the general public. This roadmap will clearly identify promising ideas for future BNCI activities that could be supported by the EU program Horizon 2020. The roadmap will display opportunities but also limitations and constraints for the industrialization and commercialization of BNCIs.

- We will investigate synergies with relevant fields. Fields that already impacted on BNCI research include assistive technologies, sensor technologies, robotics, information retrieval, neuroscience, machine learning, communication technology, human-computer interaction, ergonomics, and rehabilitation. One possible synergy from the field of machine learning could be an open database of BCI data sets and algorithms, which will increase competitiveness and reproducibility of results.

- The concepts of a user-centered design, specifically (but not exclusively) for people with motor, sensory, and cognitive disabilities will be more widespread, known, and understood.

- The involvement of industrial stakeholders throughout the duration of BNCI Horizon 2020 leads to a strong impact on a future BNCI market. For example by identifying possible products, new end user groups, and synergies with other fields. Similarly, we will actively involve end users throughout the duration of BNCI Horizon 2020, who will contribute feedback and influence the direction of our roadmap.

- BNCI Horizon 2020 will contribute to a foundation of a BCI Society through approaching key stakeholders, organization of meetings, targeted discussions, and 
evaluation of possible scenarios to implement this society. A BCI Society will have a very strong impact on the whole field, because this would be the first time that people working with BCIs could speak with a common public voice.

- A common terminology and common standards for the evaluation of BNCIs promoted by our project will lead to stronger and more efficient research efforts and collaborations. We will create ethical guidelines for the use of BNCIs, and we will seek contact with the general public and address their questions, concerns, and ideas. This will benefit all people involved with the development and the (commercial) use of BCIs, including the general public.

Acknowledgments. This work was supported by EU FP7 CSA "BNCI Horizon 2020: The Future of Brain/Neural Computer Interaction”, FP7-ICT-2013-10-609593.

\section{References}

1. Kübler, A., Neumann, N., Kaiser, J., Kotchoubey, B., Hinterberger, T., Birbaumer, N.: Brain-computer communication: self-regulation of slow cortical potentials for verbal communication. Archives of Physical Medicine and Rehabilitation 82, 1533-1539 (2001)

2. Scherer, R., Müller-Putz, G.R., Neuper, C., Graimann, B., Pfurtscheller, G.: An asynchronously controlled EEG-based virtual keyboard: improvement of the spelling rate. IEEE Transactions on Biomedical Engineering 51(6), 979-984 (2004)

3. Treder, M.S., Schmidt, N.M., Blankertz, B.: Gaze-independent brain-computer interfaces based on covert attention and feature attention. Journal of Neural Engineering 8(6), 066003 (2011)

4. Birbaumer, N., Ghanayim, N., Hinterberger, T., Iversen, I., Kotchoubey, B., Kübler, A., Perelmouter, J., Taub, E., Flor, H.: A spelling device for the paralysed. Nature 398, 297-298 (1999)

5. del R. Millán, J., Renkens, F., Mouriño, J., Gerstner, W.: Brain-actuated interaction. Artificial Intelligence 159(1-2), 241-259 (2004)

6. Müller-Putz, G.R., Scherer, R., Pfurtscheller, G., Rupp, R.: EEG-based neuroprosthesis control: a step towards clinical practice. Neuroscience Letters 382, 169-174 (2005)

7. Cincotti, F., Mattia, D., Aloise, F., Bufalari, S., Schalk, G., Oriolo, G., Cherubini, A., Marciani, M.G., Babiloni, F.: Non-invasive brain-computer interface system: towards its application as assistive technology. Brain Research Bulletin 75(6), 796-803 (2008)

8. Aloise, F., Schettini, F., Aricò, P., Leotta, F., Salinari, S., Mattia, D., Babiloni, F., Cincotti, F.: P300-based brain-computer interface for environmental control: an asynchronous approach. Journal of Neural Engineering 8(2), 025025 (2011)

9. Leeb, R., Friedman, D., Müller-Putz, G.R., Scherer, R., Slater, M., Pfurtscheller, G.: Selfpaced (asynchronous) BCI control of a wheelchair in virtual environments: a case study with a tetraplegic. Computational Intelligence and Neuroscience 79642, 1-8 (2007)

10. Galán, F., Nuttin, M., Lew, L., Ferrez, P.W., Vanacker, G., Philips, J., del R. Millán, J.: A brain-actuated wheelchair: asynchronous and non-invasive brain-computer interfaces for continuous control of robots. Clinical Neurophysiology 119, 2159-2169 (2008)

11. Andersson, P., Pluim, J.P., Viergever, M.A., Ramsey, N.F.: Navigation of a telepresence robot via covert visuospatial attention and real-time fMRI. Brain Topography 26, 177-185 (2013) 
12. Carlson, T., Tonin, L., Leeb, R., Rohm, M., Rupp, R., Al-Khodairy, A., del R. Millán, J.: BCI telepresence: a six patient evaluation. In: TOBI Workshop III: Bringing BCIs to EndUsers - Facing the Challenge, pp. 18-19. University of Würzburg (2012)

13. Mugler, E.M., Ruf, C.A., Halder, S., Bensch, M., Kübler, A.: Design and implementation of a P300-based brain-computer interface for controlling an internet browser. IEEE Transactions on Neural Systems and Rehabilitation Engineering 18(6), 599-609 (2010)

14. Zickler, C., Riccio, A., Leotta, F., Hillian-Tress, S., Halder, S., Holz, E., Staiger-Sälzer, P., Hoogerwerf, E.-J., Desideri, L., Mattia, D., Kübler, A.: A brain-computer interface as input channel for a standard assistive technology software. Clinical EEG Neuroscience (42), 236-244 (2011)

15. Krepki, R., Blankertz, B., Curio, G., Müller, K.-R.: The Berlin Brain-Computer Interface (BBCI): towards a new communication channel for online control in gaming applications. Journal of Multimedia Tools and Applications 33(1), 73-90 (2007)

16. Nijholt, A., Oude Bos, D., Reuderink, B.: Turning Shortcomings into Challenges: BrainComputer Interfaces for Games. Entertainment Computing 1(2), 85-94 (2009)

17. Plass-Oude Bos, D., Reuderink, B., van de Laar, B., Gürkök, H., Mühl, C., Poel, M., Nijholt, A., Heylen, D.: Brain-Computer Interfacing and Games. In: Tan, D., Nijholt, A. (eds.) Brain-Computer Interfaces: Applying our Minds to Human-Computer Interaction, pp. 149-178. Springer, London (2010)

18. Holz, E.M., Höhne, J., Staiger-Sälzer, P., Tangermann, M., Kübler, A.: Brain-computer interface controlled gaming: evaluation of usability by severely motor restricted end-users. Artificial Intelligence in Medicine 59(2), 111-120 (2013)

19. Zickler, C., Halder, S., Kleih, S.C., Herbert, C., Kübler, A.: Brain painting: usability testing according to the user-centered design in end users with severe motor paralysis. Artificial Intelligence in Medicine 59(2), 99-110 (2013)

20. Gürkök, H., Nijholt, A.: Affective Brain-Computer Interfaces for Arts. In: Nijholt, A., D'Mello, S., Pantic, M. (eds.) Affective Computing and Intelligent Interaction (ACII 2013), pp. 827-831. IEEE Press, New York (2013)

21. Kübler, A., Kotchoubey, B., Kaiser, J., Wolpaw, J.R., Birbaumer, N.: Brain-computer communication: unlocking the locked in. Psychological Bulletin 127, 358-375 (2001)

22. Wolpaw, J.R., Birbaumer, N., McFarland, D.J., Pfurtscheller, G., Vaughan, T.M.: Braincomputer interfaces for communication and control. Clinical Neurophysiology 113, 767791 (2002)

23. Wolpaw, J.R., Winter Wolpaw, E.: Brain-computer interfaces: something new under the sun. In: Wolpaw, J.R., Winter Wolpaw, E. (eds.) Brain-Computer Interfaces: Principles and Practice, pp. 3-12. Oxford University Press, Oxford (2012)

24. Zander, T.O., Kothe, C.: Towards passive brain-computer interfaces: applying braincomputer interface technology to human-machine systems in general. Journal of Neural Engineering 8, 025005 (2011)

25. Mühl, C., van den Broek, E.L., Brouwer, A.-M., Nijboer, F., van Wouwe, N., Heylen, D.: Multi-modal affect induction for affective brain-computer interfaces. In: D'Mello, S., Graesser, A., Schuller, B., Martin, J.-C. (eds.) ACII 2011, Part I. LNCS, vol. 6974, pp. 235-245. Springer, Heidelberg (2011)

26. Garcia Molina, G., Tsoneva, T., Nijholt, A.: Emotional Brain-Computer Interfaces. International Journal of Autonomous and Adaptive Communications Systems (IJAACS) 6(1), 9-25 (2013)

27. Ferrez, P.W., del R. Millán, J.: Error-related EEG potentials generated during simulated brain-computer interaction. IEEE Transactions on Biomedical Engineering 55, 923-929 (2008) 
28. Müller, K.-R., Tangermann, M., Dornhege, G., Krauledat, M., Curio, G., Blankertz, B.: Machine learning for real-time single-trial EEG analysis: from brain-computer interfacing to mental state monitoring. Journal of Neuroscience Methods (167), 82-90 (2008)

29. Pfurtscheller, G., Allison, B.Z., Brunner, C., Bauernfeind, G., Solis-Escalante, T., Scherer, R., Zander, T.O., Müller-Putz, G.R., Neuper, C., Birbaumer, N.: The hybrid BCI. Frontiers in Neuroscience 4(3) (2010)

30. Mueller-Putz, G.R., Breitwieser, C., Cincotti, F., Leeb, R., Schreuder, M., Leotta, F., Tavella, M., Bianchi, L., Kreilinger, A., Ramsay, A., Rohm, M., Sagebaum, M., Tonin, L., Neuper, C., del R. Millán, J.: Tools for Brain-Computer Interaction: a general concept for a hybrid BCI (hBCI). Frontiers in Neuroinformatics 5, 30 (2011)

31. Nijholt, A., Allison, B.Z., Jacob, R.K.: Brain-Computer Interaction: Can Multimodality Help? In: Bourlard, H., et al. (eds.) 13th International Conference on Multimodal Interaction, pp. 35-39. ACM, New York (2011)

32. Blankertz, B., Tangermann, M., Vidaurre, C., Fazli, S., Sannelli, C., Haufe, S., Maeder, C., Ramsey, L.E., Sturm, I., Curio, G., Müller, K.-R.: The Berlin Brain-Computer Interface: Non-Medical Uses of BCI Technology. Frontiers in Neuroscience 4, 198 (2010)

33. Kohlmorgen, J., Dornhege, G., Braun, M., Blankertz, B., Müller, K.-R., Curio, G., Hagemann, K., Bruns, A., Schrauf, M., Kincses, W.: Improving human performance in a real operating environment through real-time mental workload detection. In: Dornhege, G., del R. Millán, J., Hinterberger, T., McFarland, D., Müller, K.-R. (eds.) Toward BrainComputer Interfacing, pp. 409-422. MIT Press, Cambridge (2007)

34. Martel, A., Dähne, S., Blankertz, B.: EEG Predictors of Covert Vigilant Attention. Journal of Neural Engineering (in press, 2014)

35. Haufe, S., Treder, M.S., Gugler, M.F., Sagebaum, M., Curio, G., Blankertz, B.: EEG potentials predict upcoming emergency brakings during simulated driving. Journal of Neural Engineering 8, 056001 (2011)

36. Porbadnigk, A.K., Treder, M.S., Blankertz, B., Antons, R., Schleicher, R., Möller, S., Curio, G., Müller, K.-R.: Single-trial analysis of the neural correlates of speech quality perception. Journal of Neural Engineering 10(5), 056003 (2013)

37. Schmidt, N.M., Blankertz, B., Treder, M.S.: Online detection of error-related potentials boosts the performance of mental typewriters. BMC Neurosci. 13, 19 (2012)

38. Venthur, B., Blankertz, B., Gugler, M.F., Curio, G.: Novel Applications of BCI Technology: Psychophysiological Optimization of Working Conditions in Industry. In: Systems, Man and Cybernetics (SMC 2010), pp. 417-421. IEEE Press, New York (2010)

39. Future BNCI: A Roadmap for Future Directions in Brain/Neuronal Computer Interaction. The Future BNCI Project (2012), http://bnci-horizon-2020.eu/images/ bncih2020/FBNCI_Roadmap.pdf

40. Kübler, A., Mattia, D., Rupp, R., Tangermann, M.: Facing the challenge: brain-computer interfaces to end-users. Artificial Intelligence in Medicine 59(2), 55-60 (2013)

41. Kübler, A., Holz, E., Kaufmann, J.: A user-centered approach for bringing BCI controlled applications to end users. In: Reza-Fazel, R. (ed.) Brain-Computer Interface Systems - Recent Progress and Future Prospects. InTech, New York (2013)

42. Kübler, A., Zickler, C., Holz, E., Kaufmann, T., Riccio, A., Mattia, D.: Applying the usercentred design to evaluation of Brain-Computer Interface controlled applications. Biomedical Engineering / Biomedizinische Technik 58(suppl. 1) (2013), doi:10.1515/bmt-20134438 